\title{
When are maps preserving semi-inner products linear?
}

\author{
PAWEŁ WÓJCIK(D)
}

\begin{abstract}
We observe that every map between finite-dimensional normed spaces of the same dimension that respects fixed semi-inner products must be automatically a linear isometry. Moreover, we construct a uniformly smooth renorming of the Hilbert space $\ell_{2}$ and a continuous injection acting thereon that respects the semi-inner products, yet it is non-linear. This demonstrates that there is no immediate extension of the former result to infinite dimensions, even under an extra assumption of uniform smoothness.
\end{abstract}

Mathematics Subject Classification. Primary: 39B52, 46C50; Secondary: 46B04, 47J05, 46B20.

Keywords. Functional equation, Orthogonality equation, Semi-inner product, Uniformly smooth Banach space.

\section{Introduction}

It is an easy consequence of the polarisation identity that unitary maps between Hilbert spaces, that is, maps preserving the inner product, are automatically linear. Since inner-product spaces are characterised by the inextricable connection between the norm and the inner product, the aforementioned fact does not have a canonical interpretation in the non-Hilbertian setting. Nonetheless, natural approaches to extending Uhlhorn's version of Wigner's theorem on symmetry transformations [5] are available in the Banach-space setting, for example, in terms of Birkhoff-James orthogonality [1] or semi-inner products [3]. In the present paper we focus on the latter approach.

Lumer [4] and Giles [2] proved that reminiscences of inner products are available in arbitrary normed spaces as for every normed space $X$ one may find a pairing $[\cdot \mid \cdot]$ thereon (a semi-inner product) that assumes scalar values, is linear in the first variable, anti-homogeneous in the second variable, and the following form of the Cauchy-Schwarz inequality holds:

$$
|[x \mid y]| \leqslant\|x\| \cdot\|y\| \quad(x, y \in X)
$$


with $[x \mid x]=\|x\|^{2}$. In particular, for each $w \in X,[\cdot \mid w] \in X^{*}$.

Semi-inner products are, in general, non-unique: a normed space $X$ has a unique semi-inner product if and only if it is smooth, that is every nonzero vector $x \in X$ admits a unique norming functional, that is, a norm-one functional $\varphi_{x} \in X^{*}$ such that $\left\langle\varphi_{x}, x\right\rangle=\|x\|$. In the case where $X$ is smooth, we have

$$
[x \mid y]=\|y\| \cdot\left\langle\varphi_{y}, x\right\rangle \quad(x, y \in X) .
$$

We observed in [6, Theorem 7] that if $X$ is a non-Hilbertian finite-dimensional space with $\operatorname{dim} X \geqslant 3$ that is smooth, then there exists a space $V$ of dimension $\operatorname{dim} X-1$ and a non-linear map $f: V \rightarrow X$ that preserves semi-inner products. The map $f$ may even be discontinuous.

The first result demonstrates that for $X$ and $Y$ having equal finite dimensions, without any additional hypotheses, a semi-inner product preserving function between $X$ and $Y$ must be a linear isometry.

Theorem 1. Let $X$ and $Y$ be normed spaces with fixed semi-inner products $[\cdot \mid \cdot]_{X}$ and $[\cdot \mid \cdot]_{Y}$, respectively. Suppose that $f: X \rightarrow Y$ is a function such that

$$
[f(x) \mid f(y)]_{Y}=[x \mid y]_{X} \quad(x, y \in X)
$$

If either

(a) $X$ and $Y$ have the same finite dimension, or

(b) $X$ has a Schauder basis $\left(e_{i}\right)$ and $\left(f\left(e_{i}\right)\right)$ is a Schauder basis of $Y$, then $f$ is a linear isometry.

The proof will be presented in the subsequent section. We highlight Theorem 1 as clause (a) appears to be optimal in the case where no further assumptions on $f$ are imposed in the light of the following blatant counterexample of an analogous statement in infinite dimensions.

Theorem 2. There exists a uniformly smooth renorming $X$ of the Hilbert space $\ell_{2}$ and a non-linear injection $f: X \rightarrow X$ such that

$$
[f(x) \mid f(y)]=[x \mid y] \quad(x, y \in X) .
$$

Moreover, $f$ may be chosen to be either continuous or discontinuous.

Since $X$ is smooth, the choice of the semi-inner product is unambiguous. Regrettably, Theorem 2 refutes a side result from a recent paper by Ilišević and Turnšek [3, Proposition 2.4(ii)], where it was claimed that if $X$ is a smooth Banach space and $f: X \rightarrow X$ is a (possibly non-surjective) map satisfying (3), then $f$ is necessarily a linear isometry. Their proof contains a flaw as explained in Remark 6. However, the main results in [3] are dealing with surjective functions between smooth normed spaces which satisfy the Wigner equation. It can be easily verified (see [3, Proposition 2.4(i)]) that, even in arbitrary normed 
spaces, the additional assumption of surjectivity forces semi-inner product preserving functions to be linear.

Our notation and terminology are standard. We consider normed spaces over the field $\mathbb{K}$ of real or complex numbers. A normed space $X$ is strictly convex if the unit sphere of $X$ does not contain non-trivial line segments. We denote by $\langle\cdot, \cdot\rangle$ the duality pairing between a normed space $X$ and its dual $X^{*}$. When $X$ is an inner-product space, we denote by $\langle\cdot \mid \cdot\rangle$ the underlying inner product. A normed space is uniformly smooth, if for every $\varepsilon>0$ there exists $\delta>0$ such that if $x, y \in X$ are vectors such that $\|x\|=1$ and $\|y\| \leqslant \delta$ then $\|x+y\|+\|x-y\| \leqslant 2+\varepsilon\|y\|$. Uniformly smooth spaces are, in particular, smooth. For the sake of completeness, we record the following simple property of smooth spaces.

Lemma 3. Let $X$ be a smooth normed space. If $u, w \in X$ are non-zero vectors and $\|u+w\|=\|u\|+\|w\|$, then $\varphi_{u}=\varphi_{w}$.

Proof. Let $u, w \in X$ be vectors with $\|u+w\|=\|u\|+\|w\|$. It is clear that $\|u+w\|=\varphi_{u+w}(u+w)$. So, it follows that

$$
\begin{aligned}
\|u\|+\|w\| & =\|u+w\|=\varphi_{u+w}(u+w)=\varphi_{u+w}(u)+\varphi_{u+w}(w) \\
& \leqslant\left|\varphi_{u+w}(u)+\varphi_{u+w}(w)\right| \leqslant\left|\varphi_{u+w}(u)\right|+\left|\varphi_{u+w}(w)\right| \\
& \leqslant\|u\|+\|w\| .
\end{aligned}
$$

Thus $\varphi_{u+w}(u)+\varphi_{u+w}(w)=\|u\|+\|w\|$. Moreover, we know that $\left|\varphi_{u+w}(u)\right| \leqslant$ $\|u\|$ and $\left|\varphi_{u+w}(w)\right| \leqslant\|w\|$. This clearly forces $\varphi_{u+w}(u)=\|u\|$ and $\varphi_{u+w}(w)=$ $\|w\|$. On the other hand, both $\varphi_{u}(u)=\|u\|$ and $\varphi_{w}(w)=\|w\|$ hold. Since $X$ is smooth, we get $\varphi_{u+w}=\varphi_{u}$ and $\varphi_{u+w}=\varphi_{w}$. Hence $\varphi_{u}=\varphi_{w}$.

\section{Proofs of Theorems 1 and 2}

We start by proving Theorem 1 . For the sake of brevity, we shall use the symbol $[\cdot \mid \cdot]$ for (fixed) semi-inner products both in $X$ and $Y$, hoping it will not lead to unnecessary confusion. In order to prove the theorem, it suffices to show that $f$ is linear.

Proof of Theorem 1. We will prove clause (b) first. Suppose that $\left(e_{i}\right)$ is a Schauder basis of $X$ and $\left(f\left(e_{i}\right)\right)$ is a Schauder basis of $Y$. The proof of the case where $X$ is finite-dimensional (so that a Schauder basis is just an ordinary algebraic basis) is mutatis mutandis the same, so we will keep writing infinite series bearing in mind that the proof works equally well for the finite-dimensional case with $\infty$ replaced by $\operatorname{dim} X$ ).

We will show that for any scalars $\beta_{1}, \beta_{2}, \ldots$

$$
f\left(\sum_{i=1}^{\infty} \beta_{i} e_{i}\right)=\sum_{i=1}^{\infty} \beta_{i} f\left(e_{i}\right)
$$


as long as the series $\sum_{i=1}^{\infty} \beta_{i} e_{i}$ converges in $X$.

Fix $x \in X$. Since $\left(f\left(e_{i}\right)\right)_{i=1}^{\infty}$ is a basis, there are uniquely determined scalars $\beta_{1}, \beta_{2}, \ldots \in \mathbb{K}$ such that

$$
f(x)=\sum_{i=1}^{\infty} \beta_{i} f\left(e_{i}\right)
$$

Let $x_{m}=\sum_{i=1}^{m} \beta_{i} e_{i}$. It is enough to show that $x_{m} \rightarrow x$ as $m \rightarrow \infty$. Let us define the numbers $\varepsilon_{m}:=\left\|f(x)-\sum_{i=1}^{m} \beta_{i} f\left(e_{i}\right)\right\|$. Clearly, $\varepsilon_{m} \rightarrow 0$ as $m \rightarrow \infty$. For every unit vector $u \in X$, we have $1=\|u\|=\|f(u)\|$. Thus, for every $m$ we have

$$
\left|\left[f(x)-\sum_{i=1}^{m} \beta_{i} f\left(e_{i}\right) \mid f(u)\right]\right| \leqslant\left\|f(x)-\sum_{i=1}^{m} \beta_{i} f\left(e_{i}\right)\right\| \cdot\|f(u)\| \leqslant \varepsilon_{m} \cdot 1 .
$$

Using the linearity of semi-inner products in the first variable, it follows that $\left|[f(x) \mid f(u)]-\left[\sum_{i=1}^{m} \beta_{i} f\left(e_{i}\right) \mid f(u)\right]\right|=\left|[f(x) \mid f(u)]-\sum_{i=1}^{m} \beta_{i}\left[f\left(e_{i}\right) \mid f(u)\right]\right| \leqslant \varepsilon_{m}$.

Combining the above inequality with (2) yields

$$
\left|[x \mid u]-\sum_{i=1}^{m} \beta_{i}\left[e_{i} \mid u\right]\right| \leqslant \varepsilon_{m} .
$$

Consequently,

$$
\left|\left[x-\sum_{i=1}^{m} \beta_{i} e_{i} \mid u\right]\right| \leqslant \varepsilon_{m}
$$

which means that $\left|\left[x-x_{m} \mid u\right]\right| \leqslant \varepsilon_{m}$. Since $\left\{[\cdot \mid w] \in X^{*}:\|w\|=1, w \in X\right\}$ is a 1-norming subset in the dual ball of $X^{*}$, we can conclude that we have $\left\|x-x_{m}\right\| \leqslant \varepsilon_{m}$, so $x_{m} \rightarrow x$. We have thus proved that $f\left(\sum_{i=1}^{\infty} \beta_{i} e_{i}\right)=$ $\sum_{i=1}^{\infty} \beta_{i} f\left(e_{i}\right)$. In particular, $f$ is linear, hence also isometric because it preserves the semi-inner products.

Now, in order to prove clause (a), it is enough to show that $f$ maps linearly independent sets to linearly independent sets.

Let $n=\operatorname{dim} X$. Fix a basis $\left\{b_{1}, \ldots, b_{n}\right\}$ for $X$. We claim that the set $\left\{f\left(b_{1}\right), \ldots, f\left(b_{n}\right)\right\}$ is linearly independent in $Y$. To see this, suppose that $\sum_{k=1}^{n} \alpha_{k} f\left(b_{k}\right)=0$. It follows from (2) that

$$
\begin{aligned}
\left\|\sum_{k=1}^{n} \alpha_{k} b_{k}\right\|^{2} & =\left[\sum_{k=1}^{n} \alpha_{k} b_{k} \mid \sum_{k=1}^{n} \alpha_{k} b_{k}\right]=\sum_{k=1}^{n} \alpha_{k}\left[b_{k} \mid \sum_{k=1}^{n} \alpha_{k} b_{k}\right] \\
& =\sum_{k=1}^{n} \alpha_{k}\left[f\left(b_{k}\right) \mid f\left(\sum_{k=1}^{n} \alpha_{k} b_{k}\right)\right]
\end{aligned}
$$




$$
\begin{aligned}
& =\left[\sum_{k=1}^{n} \alpha_{k} f\left(b_{k}\right) \mid f\left(\sum_{k=1}^{n} \alpha_{k} b_{k}\right)\right] \\
& =\left[0 \mid f\left(\sum_{k=1}^{n} \alpha_{k} b_{k}\right)\right]=0 .
\end{aligned}
$$

Hence $\sum_{k=1}^{n} \alpha_{k} b_{k}=0$. Since the vectors $b_{1}, \ldots, b_{n}$ are linearly independent, we have $\alpha_{1}=\ldots=\alpha_{n}=0$. This means that the vectors $f\left(b_{1}\right), \ldots, f\left(b_{n}\right)$ are linearly independent too. Consequently, $\left\{f\left(b_{1}\right), \ldots, f\left(b_{n}\right)\right\}$ is a basis for $Y$. Thus we may apply (b), and the proof is complete.

Before we prove Theorem 2, we need to introduce the main building block that we shall use to construct the sought renorming of $\ell_{2}$.

Let $\left(Z,\|\cdot\|_{o}\right)$ be a two-dimensional normed space that is smooth but not strictly convex. Then there are distinct vectors $u, w \in Z$ such that the line segment joining $u$ and $w$ lies in the unit sphere $S_{Z}$ of $Z$. Without loss of generality, we may assume that $Z=\mathbb{K}^{2}$ as a vector space and $u=(-c, 1)$, $w=(c, 1)$ for some real number $0<c<1$. Thus $(0,1) \in S_{Z}$. Moreover, without loss of generality we may assume that $(1,0) \in S_{Z}$.

Lemma 4. Let $x_{1} \in \mathbb{K}$ and $\eta \in(0, c)$. Then $\left\|\left(\eta x_{1}, x_{1}\right)\right\|_{o}=\left|x_{1}\right|$.

Proof. Since $0<\eta<c$, we have $\frac{\eta+c}{2 c} \in[0,1]$ and

$$
(\eta, 1)=\left(1-\frac{\eta+c}{2 c}\right) u+\frac{\eta+c}{2 c} w \in \operatorname{conv}\{u, w\} \subseteq S_{Z} .
$$

Thus $(\eta, 1) \in S_{Z}$, i.e., $\|(\eta, 1)\|_{o}=1$. Since $(0,1) \in S_{Z},\|(0,1)\|_{o}=1$. Therefore $\left\|\left(\eta x_{1}, x_{1}\right)\right\|_{o}=\left|x_{1}\right| \cdot\|(\eta, 1)\|_{o}=\left|x_{1}\right| \cdot 1=\left|x_{1}\right|$.

Proof of Theorem 2. We shall consider the space $X=\mathbb{K} \oplus_{2} \ell_{2}(Z)$, the $\ell_{2}$-sum of infinitely many copies of $Z$ and the one-dimensional space. The norm in $X$ is thus given by

$$
\|x\|:=\sqrt{\left|x_{1}\right|^{2}+\sum_{k=1}^{\infty}\left\|\left(x_{2 k}, x_{2 k+1}\right)\right\|_{o}^{2}},
$$

where $x=\left(x_{1},\left(x_{2}, x_{3}\right),\left(x_{4}, x_{5}\right),\left(x_{6}, x_{7}\right), \ldots\right) \in X$.

The space $X$ is uniformly smooth because $Z$ is smooth (uniformly smooth as it is finite-dimensional) and uniform smoothness passes to $\ell_{2}$-sums of infinitely many copies of a uniformly smooth space [7, Corollary 4.9]. Since $Z$ is isomorphic to the two-dimensional Hilbert space, $X$ is isomorphic to $\ell_{2}\left(\ell_{2}^{2}\right)$, which is isometric to $\ell_{2}$.

For a number $\eta \in(0, c)$, let $h_{\eta}: X \rightarrow X$ be a linear map given by

$$
h_{\eta}\left(x_{1},\left(x_{2}, x_{3}\right),\left(x_{4}, x_{5}\right), \ldots\right):=\left(0,\left(\eta x_{1}, x_{1}\right),\left(x_{2}, x_{3}\right),\left(x_{4}, x_{5}\right), \ldots\right) .
$$


Applying Lemma 4 to (4) we deduce that $h_{\eta}$ is a linear isometry. Consequently, as in $[3$, Corollary 2.5 (ii) $\Rightarrow($ i) $]$,

$$
\left[h_{\eta}(x) \mid h_{\eta}(y)\right]=[x \mid y] \quad(x, y \in X, \eta \in(0, c)) .
$$

Combining (1) with (6) we may rearrange (6) as

$$
\left\|h_{\eta}(y)\right\|_{o} \cdot\left\langle\varphi_{h_{\eta}(y)}, h_{\eta}(x)\right\rangle=[x \mid y] \quad(x, y \in X, \eta \in(0, c)) .
$$

Moreover, putting $y$ in place of $x$ in (6) we get

$$
\left\|h_{\eta}(y)\right\|_{o}=\|y\|_{o} \quad(\eta \in(0, c)) .
$$

We are now ready to construct the sought non-linear map that preserves semi-inner products.

For this, we fix a function $\gamma:[0, \infty) \rightarrow[0, \infty)$ with $\gamma(0)=0$ such that $0<\gamma(t)<c(t \in(0, \infty))$ and $\gamma$ is not constant on $(0, \infty)$. Next we choose a function $\eta: X \rightarrow[0, c)$ by

$$
\eta(x):=\gamma(\|x\|) \quad(x \in X)
$$

Then, we define a map $f: X \rightarrow X$ by the formula

$$
f\left(x_{1},\left(x_{2}, x_{3}\right),\left(x_{4}, x_{5}\right), \ldots\right):=\left(0,\left(\eta(x) x_{1}, x_{1}\right),\left(x_{2}, x_{3}\right),\left(x_{4}, x_{5}\right), \ldots\right) .
$$

Then we may recognise that

$$
f(x)=h_{\eta(x)}(x) \quad(x \in X) .
$$

Consequently, $f$ fails to be linear. However, in the case where

- $\gamma$ is continuous and non-constant on $(0, \infty), f$ is continuous;

- $\gamma$ is discontinuous on $(0, \infty), f$ is discontinuous too.

We claim that for all $x, y \in X$ we have $[f(x) \mid f(y)]=[x \mid y]$. For this, fix $x, y \in X$ and consider the associated maps $h_{\eta(y)}, h_{\eta(x)}: X \rightarrow X$. Applying again Lemma 4 to (4) and (5), we conclude that

$$
\left\|h_{\eta(y)}(y)+h_{\eta(x)}(y)\right\|_{o}=\left\|h_{\eta(y)}(y)\right\|_{o}+\left\|h_{\eta(x)}(y)\right\|_{o} .
$$

It follows from Lemma 3 that $\varphi_{h_{\eta(y)}(y)}=\varphi_{h_{\eta(x)}(y)}$, i.e.,

$$
\left\langle\varphi_{h_{\eta(y)}(y)}, w\right\rangle=\left\langle\varphi_{h_{\eta(x)}(y)}, w\right\rangle \quad(w \in X) .
$$

Consequently,

$$
\begin{aligned}
{[f(x) \mid f(y)] } & \stackrel{(1)}{=}\|f(y)\|_{o} \cdot\left\langle\varphi_{f(y)}, f(x)\right\rangle \stackrel{(9)}{=}\left\|h_{\eta(y)}(y)\right\|_{o} \cdot\left\langle\varphi_{h_{\eta(y)}(y)}, h_{\eta(x)}(x)\right\rangle \\
& \stackrel{(10)}{=}\left\|h_{\eta(y)}(y)\right\|_{o} \cdot\left\langle\varphi_{h_{\eta(x)}(y)}, h_{\eta(x)}(x)\right\rangle \\
& \stackrel{(8)}{=}\|y\|_{o} \cdot\left\langle\varphi_{h_{\eta(x)}(y)}, h_{\eta(x)}(x)\right\rangle \\
& \stackrel{(8)}{=}\left\|h_{\eta(x)}(y)\right\|_{o} \cdot\left\langle\varphi_{h_{\eta(x)}(y)}, h_{\eta(x)}(x)\right\rangle \stackrel{(7)}{=}[x \mid y] .
\end{aligned}
$$

This shows that $f: X \rightarrow X$ is indeed a non-linear map preserving semi-inner products. 
Remark 5. In the above construction one may consider the $\ell_{p}$-sums for $p \in$ $(1, \infty)$ instead of the $\ell_{2}$-sum. This will lead to a renorming of $\ell_{p}$ on which one may find a non-linear injection preserving the (unique) semi-inner products.

Remark 6. In the proof of [3, Proposition 2.4], the authors postulated the following inclusion:

$$
\left\{\xi \varphi_{f(z)} \in X^{*}: z \in X, \xi \in \mathbb{C}\right\} \supseteq\left\{\xi \varphi_{f(z)} \circ f \in X^{*}: z \in X, \xi \in \mathbb{C}\right\},
$$

where $\varphi_{f(z)} \circ f=\varphi_{z}$ (see [3, p. 1265, third line from the bottom]), which fails already in the Hilbert-space setting.

To see this, let us consider the Hilbert space $\ell_{2}$. The only semi-inner product on $\ell_{2}$ is the inner product $\langle\cdot \mid \cdot\rangle$ itself. We consider the unilateral shift on $\ell_{2}$, which is a non-surjective isometry $f: \ell_{2} \rightarrow \ell_{2}$; it is given by the formula

$$
f(x)=f\left(x_{1}, x_{2}, x_{3}, \ldots\right)=\left(0, x_{1}, x_{2}, x_{3}, \ldots\right) \quad\left(x=\left(x_{j}\right)_{j=1}^{\infty} \in \ell_{2}\right) .
$$

It is easy to see that $\langle f(x) \mid f(y)\rangle=\langle x \mid y\rangle$ and for $z \in \ell_{2} \backslash\{0\}$ we have

$$
\xi\left(\varphi_{f(z)} \circ f\right)(\cdot) \stackrel{(1)}{=} \frac{\xi}{\|f(z)\|}\langle f(\cdot) \mid f(z)\rangle=\frac{\xi}{\|z\|}\langle\cdot \mid z\rangle .
$$

Therefore $\left\{\xi \varphi_{f(z)} \circ f \in\left(\ell_{2}\right)^{*}: z \in \ell_{2}, \xi \in \mathbb{C}\right\}=\left(\ell_{2}\right)^{*}$. On the other hand,

$$
\begin{aligned}
& \left\{\xi \varphi_{f(z)} \in\left(\ell_{2}\right)^{*}: z \in \ell_{2}, \xi \in \mathbb{C}\right\} \\
& =\left\{\frac{\xi}{\|f(z)\|}\langle\cdot \mid f(z)\rangle \in\left(\ell_{2}\right)^{*}: z \in \ell_{2}, \xi \in \mathbb{C}\right\} \\
& =\left\{\langle\cdot \mid f(w)\rangle \in\left(\ell_{2}\right)^{*}: w \in \ell_{2}\right\} \\
& =\left\{\langle\cdot \mid w\rangle \in\left(\ell_{2}\right)^{*}: w=\left(0, w_{1}, w_{2}, \ldots\right) \in \ell_{2}\right\} \nsupseteq\left(\ell_{2}\right)^{*}
\end{aligned}
$$

which refutes (11).

\section{Acknowledgements}

The author acknowledges Professor Tomasz Kania for his collaboration in manufacturing the example of the Banach space and his supporting in editing this paper.

Open Access. This article is licensed under a Creative Commons Attribution 4.0 International License, which permits use, sharing, adaptation, distribution and reproduction in any medium or format, as long as you give appropriate credit to the original author(s) and the source, provide a link to the Creative Commons licence, and indicate if changes were made. The images or other third party material in this article are included in the article's Creative Commons licence, unless indicated otherwise in a credit line to the material. If material is not included in the article's Creative Commons licence and your intended use is not permitted by statutory regulation or exceeds the permitted use, you will need to obtain 
permission directly from the copyright holder. To view a copy of this licence, visit http:// creativecommons.org/licenses/by/4.0/.

Publisher's Note Springer Nature remains neutral with regard to jurisdictional claims in published maps and institutional affiliations.

\section{References}

[1] Blanco, A., Turnšek, A.: On maps that preserve orthogonality in normed spaces. Proc. R. Soc. Edinb. Sect. A Math. 136(4), 709-716 (2006)

[2] Giles, J.R.: Classes of semi-inner-product spaces. Trans. Am. Math. Soc. 129, 436-446 (1967)

[3] Ilišević, D., Turnšek, A.: On Wigner's theorem in smooth normed spaces. Aequat. Math. 94, 1257-1267 (2020)

[4] Lumer, G.: Semi-inner-product spaces. Trans. Am. Math. Soc. 100, 29-43 (1961)

[5] Uhlhorn, U.: Representation of symmetry transformations in quantum mechanics. Ark. Fys. 23, 307-340 (1963)

[6] Wójcik, P.: On an orthogonality equation in normed spaces. Funct. Anal. Appl. 52(3), 224-227 (2018)

[7] Zachariades, T.: On $\ell_{\psi}$-spaces and infinite $\psi$-direct sums of Banach space. Rocky Mt. J. Math. 41(3), 971-997 (2011)

Paweł Wójcik

Institute of Mathematics

Pedagogical University of Cracow

Podchorążych 2

30-084 Kraków

Poland

e-mail: pawel.wojcik@up.krakow.pl

Received: March 27, 2021

Revised: June 18, 2021

Accepted: June 18, 2021 